\title{
Caractérisation physicochimique et microbiologique des différents substrats organiques au cours de la fermentation méthanique
}

\author{
Mohamed Elamin AFILAL, Nadia BELKHADIR \\ Université Mohamed Premier, Laboratoire de biologie des plantes et microorganismes BP: 524, 60000 Oujda \\ afilalamin@yahoo.fr
}

\section{Résumé}

L'objectif de ce travail est de chercher les conditions optimales pour la production du biogaz à partir des déchets organiques notamment les résidus des plantes aromatiques et médicinales (PAM). Le biogaz est le résultat de la digestion anaérobie des matières organiques. En effet, la production du méthane à partir de la biomasse suit le principe de la fermentation dans le digesteur appelé aussi bioréacteur ou méthaniseur. La première étape est l'hydrolyse de la matière organique présente sous forme de grosses molécules, solubles ou non, comme la cellulose. Ces polymères sont cassés en monomères par des microorganismes, et les bactéries méthanogènes utilisent ensuite, les précurseurs formés, pour produire du méthane. Ces étapes biochimiques et microbiologiques se déroulent simultanément ou séparément suivant le procédé anaérobie utilisé mais à des vitesses différentes. Dans ce travail, nous avons entrepris une série d'expériences, pour fermenter les résidus des PAM et produire du biogaz inflammable. Les résultats obtenus nous permettent de tirer les conclusions suivantes :

- Les PAM restent exploitables après l'hydro-distillation, et les résidus secs restent biodégradables.

- L'optimisation et l'accélération du processus de méthanisation, nécessite le conditionnement et l'adaptation d'une culture microbienne active avec les différents substrats.

Ce travail ouvre de nouveaux horizons pour la recherche d'une alternative du développement dans le monde rural, et propose une source d'énergie renouvelable permettant à la fois, une meilleure gestion des déchets organiques, la production du gaz inflammable et aussi une boue fertilisante pour l'amendement des sols. Pour pouvoir achever ce travail, d'autres expériences sont prévues en perspective. Elles seront consacrées à la conception d'un fermenteur opérationnel dans les conditions du monde rurale.

Mots-clés : biogaz, résidus, fermentation, déchets organiques, plantes aromatiques et médicinales (PAM).

Key-words: biogas, residues, fermentation, organic wastes, aromatic and medicinal plant (PAM).

\section{Introduction}

Parmi les différentes sources énergétiques renouvelables utilisées, figure celle de la biomasse, qui constitue un gisement en continuelle augmentation et présente une très large diversité de composition. Son exploitation pour la production d'énergie, est d'autant plus intéressante qu'elle intervient également dans la préservation de l'environnement (Afilal, 2007).

L’objectif de ce travail est de tester l'efficacité d'un ferment microbien, sélectionné et entretenu au laboratoire de biologie des plantes et microorganismes (Université Mohamed $1^{\mathrm{er}}$, Oujda), pour la production de biogaz, à partir des déchets organiques, notamment les résidus des plantes aromatiques et médicinales. Le biogaz est le résultat de la méthanisation, c'est-àdire de la digestion microbienne des matières organiques en anaérobiose. Le processus biologique de la méthanisation bien qu'il soit complexe, peut se diviser en quatre étapes 
principales réparties entre différents groupes de bactéries: hydrolyse, fermentation (acidogenèse), acétogenèse et méthanogenèse.

La première étape est l'hydrolyse de la matière organique, comme les protéines, les graisses, la cellulose et l'amidon... Ces polymères sont cassés en monomères, comme les acides aminés, les acides gras et les sucres simples.

La deuxième étape est l'œuvre de bactéries acidogènes qui transforment le substrat en un mélange d'acides (lactique, butyrique, etc...) et de composés neutres (éthanol).

Lors de la troisième étape un groupe de bactéries transforme ensuite les divers acides et les autres produits issus de l'étape précédente en éléments précurseurs du méthane: acide acétique, dioxyde de carbone $\left(\mathrm{CO}_{2}\right)$ et hydrogène $\left(\mathrm{H}_{2}\right)$. Un transfert efficace de l'hydrogène des bactéries acétogènes aux bactéries méthanogènes est indispensable pour assurer le bon déroulement de l'ensemble du processus de méthanisation.

Enfin durant la quatrième étape, les bactéries méthanogènes utilisent ensuite les précurseurs formés lors des étapes précédentes pour produire du méthane.

Ces quatre étapes se déroulent simultanément ou séparément suivant le procédé anaérobie utilisé mais à des vitesses différentes. La méthanisation est réalisée par un groupe particulier de micro-organismes, les Archéobactéries. Ces micro-organismes sont distribués dans la nature, et on les trouve au niveau du tube digestif des ruminants en particulier.

Tout en créant de l'énergie, la biométhanisation produit un engrais sans odeur et de bonne qualité pour l'enrichissement du sol. (Ghaly et al. 2000, Afilal 2007). La matière organique utilisable pour la production de biogaz, comprend la matière végétale, les effluents de l'élevage et les déchets des activités industrielles et domestiques.

$\mathrm{Au}$ Maroc et surtout dans les zones rurales, plusieurs coopératives d'exploitation des PAM se retrouvent avec des quantités énormes de résidus, après distillation, et cherchent une solution pour une meilleure valorisation. Ceci nous a poussé à proposer une alternative prometteuse, qui est la valorisation par fermentation méthanique des résidus de la matière organique, que nous comptons développer, adapter et optimiser pour encourager les coopératives des PAM à augmenter leur rendement et assurer un meilleur développement de leur secteur.

\section{Matériel et méthodes}

\section{Matériel}

- Cuves de fermentation: Bouteilles en plastique transparent de 5 litres.

- Matière première (déchet de vache $V$, mélange de laboratoire L. résidus de PAM substrat P).

- Bain-marie pour assurer une température bien constante et une agitation continue.

- Des tuyaux à gaz transparents.

- Chambre à air pour piéger le gaz.

- Manomètre à eau.

- Étuve à $105^{\circ} \mathrm{C}$ et four à $600^{\circ} \mathrm{C}$.

\section{Méthodes}

\section{Préparation de la matière première}

La matière première des différents substrats est pesée et homogénéisée dans de l'eau distillée par un broyeur électrique Ultraturax à 20000t/min pendant $10 \mathrm{~min}$. Ce qui permet d'obtenir des mélanges (V, L, P) de $20 \%$ de la matière organique :

- Mélange V: bouse de vache amenée au laboratoire le jour du lancement de la fermentation. 
- Mélange L : sorte de levain microbien adapté aux déchets organiques. Il est obtenu suite à une série de repiquage d'une culture microbienne mixte, en présence de la bouse de vache et des souches, préalablement isolée au laboratoire de microbiologie, douées d'une forte activité cellulolytique.

- Mélange P : mélange de feuilles de PAM obtenu d'une coopérative de la région d'AlHoceima, traitant surtout thym, origan, lavande et ciste. Ces résidus étaient abandonnés à l'air libre plusieurs jours après avoir subit une hydrodistilllation pour en extraire les huiles essentielles. Ce mélange est débarrassé manuellement des résidus difficiles à broyer (environ 30\%).

\section{Caractérisation des substrats}

L’humidité de chaque mélange, est mesurée le plus rapidement possible, $100 \mathrm{~g}$ de chaque substrat, sont mis à l'étuve à $105 \pm 2{ }^{\circ} \mathrm{C}$ pendant 24 heures selon la norme NF M03002 (Igoud et al., 2002).

Pour la mesure de la teneur en matière organique (MO), une dizaine de grammes de chaque substrat séché et broyé, est calcinée à $550{ }^{\circ} \mathrm{C}$ pendant 4 heures au four Volca Prolabo (Igoud et al., 2002).

Les analyses sont doublées. La teneur en matière organique est obtenue par différence de pesée entre la masse du substrat sec et la masse calcinée.

Taux de MO $(\%)=\frac{\text { masse de l'échantillon sec }- \text { masse de l'échantillon calciné }}{\text { masse de l'échantillon } \mathrm{sec}} \times 100$

\section{Évaluation du biogaz produit}

Le biogaz est un mélange de plusieurs gaz, à des proportions variables. L’inflammation du biogaz, indique la présence du méthane dans le mélange.

L’évaluation de la quantité de biogaz était faite par piégeage dans une chambre à air, et/ou par un manomètre à eau.

\section{Préparation et lancement de la fermentation}

Une première expérience visait à comparer la fermentation des différents substrats, ceci à été mené dans des fermenteurs de 5 litres, les bouteilles sont remplies à $3 / 4$ par chaque mélange $(\mathrm{V}, \mathrm{L}, \mathrm{P})$, fermées et branchées à une chambre à air, puis mises dans un Bain Mari à une température de $41^{\circ} \mathrm{C}$ et une agitation moyenne continue. (Photos 1 et 2).

Pendant la fermentation, on contrôle tous les paramètres (température, agitation, temps de séjours, etc.), et toutes les observations sont notées au cours du temps.

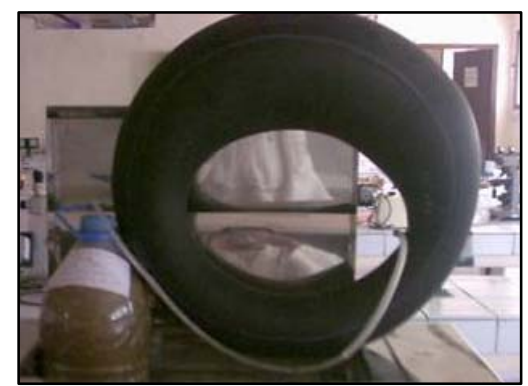

Photo 1. Production rapide du biogaz par fermentation du mélange $\left(\mathrm{V}+\mathrm{L}^{+}\right.$ P) pendant $100 \mathrm{~h}$ avec agitation continue et à $41^{\circ} \mathrm{C}$.

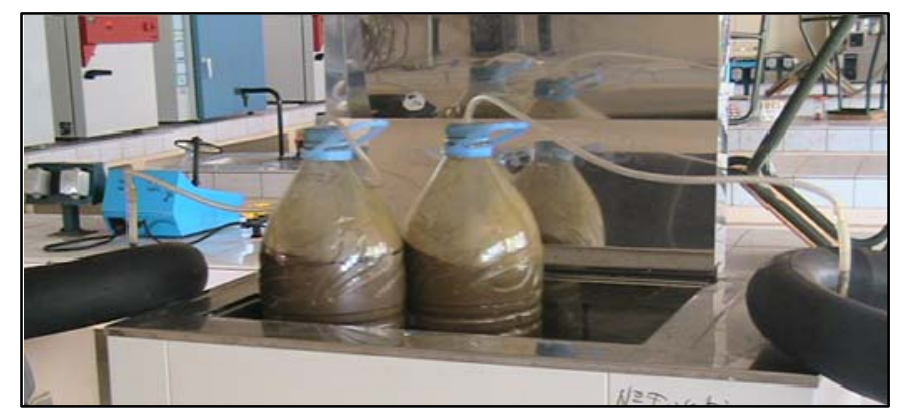

Photo 2. Production du biogaz par fermentation du mélange (V seule à droite et $\mathrm{V}+\mathrm{P}$ à gauche) pendant $100 \mathrm{~h}$ avec agitation continue et à $41^{\circ} \mathrm{C}$. 


\section{Résultats et discussion}

Dans ce travail, il a été démontré que les résidus des PAM, restent valorisables par fermentation méthanique.

L'enrichissement du milieu de culture par une flore active mixte, permettra d'accélérer le processus et le substrat organique est fermenté malgré qu'il soit essentiellement de la cellulose. Ces résultats ouvrent un nouvel horizon pour une solution alternative permettant une meilleure valorisation des résidus de PAM et une meilleure gestion des déchets organiques.

Les résultats présentés dans les tableaux 1 et 2, indiquent que la fermentation des différents substrats testés, génère du biogaz inflammable, donc riche en méthane. Ces résultats montrent également que la vitesse et la quantité de biogaz produite est beaucoup plus importante dans le cas du mélange « $\mathrm{P}+\mathrm{L}+\mathrm{V}$ » par rapport aux autres mélanges (photos 1 et 2). La biomethanisation des résidus de PAM, serait favorisée par plusieurs paramètres, d'une part le $\mathrm{pH}$ de départ est acide, se qui accélérerait la première phase d'hydrolyse, permettant ensuite un passage rapide à la phase de méthanisation par une flore plus particulière, la zone favorable à la bio-méthanisation étant entre $\mathrm{pH}$ 6,6 et $\mathrm{pH}$ 8. (Ghaly et al., 2000),. D’autre part les plantes médicinales (humidité : 7\%), sont des déchets organiques riches en cellulose (source de carbone pour les bactéries), car plus la charge organique est grande, plus la production du biogaz est rapide.

Le levain microbien $(\mathrm{L})$ accélère la production de gaz, le substrat $(\mathrm{P})$ à base de résidus de plantes aromatiques et médicinales, serait plus facilement fermentescible avec l'addition des bouses de vaches (V) et du levain (L).

Tableau 1. Caractéristiques des différents substrats avant fermentation.

\begin{tabular}{lccc}
\hline Substrats & $\begin{array}{c}\text { résidus de PAM } \\
\text { (Mélange P) }\end{array}$ & $\begin{array}{c}\text { Culture microbienne mixte sur des } \\
\text { déchets organiques (Mélange L) }\end{array}$ & $\begin{array}{c}\text { bouse de vache } \\
\text { (Mélange V) }\end{array}$ \\
\hline pH & 6,31 & 4,91 & 6.81 \\
Taux d'humidité & $7 \%$ & $11 \%$ & $6 \%$ \\
Matière minérale & $8 \%$ & $8 \%$ & $8 \%$ \\
Matière organique & $85 \%$ & $81 \%$ & $86 \%$ \\
\hline
\end{tabular}

Tableau 2. Fermentation des différents substrats à $41^{\circ} \mathrm{C}$ avec agitation continue dans un volume total de 4.5 litres. *Estimation par observation de la chambre à gaz.

\begin{tabular}{cccc}
\hline $\begin{array}{c}\text { Nombre de répétition des } \\
\text { expériences }\end{array}$ & $\begin{array}{c}\text { Substrats soumises à la } \\
\text { fermentation }\end{array}$ & $\begin{array}{c}\text { Production de gaz en } \\
\text { 2 jours* }\end{array}$ & $\begin{array}{c}\text { Production de gaz en } \\
\mathbf{4} \text { jours* }\end{array}$ \\
\hline 2 & $\mathrm{~V}$ & - & + \\
3 & $\mathrm{~L}+\mathrm{P}$ & + & + \\
4 & $\mathrm{~L}+\mathrm{P}+\mathrm{V}$ & + & ++++ \\
6 & $\mathrm{~V}+\mathrm{P}$ & - & + \\
\hline
\end{tabular}

\section{Conclusion}

Dans les prochains essais, il est envisageable d'installer des fermenteurs de plus grande dimension et entreprendre des mesures de caractérisation : taux de production de gaz par unité de temps, taux de $\mathrm{CH}_{4}$ et des autres composants du gaz produit, temps de rétention de la matière organique......pour cela nous feront usage d'instruments de mesures précises (pHmètre, thermomètre, debimètre, mesure de la DCO........). 
Sachant que le constituant principal des résidus des PAM est la cellulose, qui est un substrat dont la décomposition se fait essentiellement par une variété de microorganismes telluriques, il est envisageable, dans l'avenir, de mener d'autres expériences avec un enrichissement en bactéries cellulolytiques dans la première phase de fermentation, pour accélérer le processus et favoriser l'installation rapide de la phase de méthanisation.

L’évaluation de la biométhanisation des résidus, devrait aussi être étudiée pour chaque PAM séparément.

\section{Remerciements}

Ce travail est financé par la CUD de Belgique dans le cadre du projet de collaboration avec l'Université Mohamed $1^{\mathrm{er}}$ Oujda "Projet appui à la recherche scientifique P2 de 2004-2008”.

\section{Références :}

Afilal ME, Auriol M, Filali-Meknassi Y (2007) Bilan sur les sources d'énergies renouvelables au Maroc. In UnESCO, Les énergies renouvelables au Maroc - Le débat est lancé, Energie de biomasse, ISBN 9954-8068-2-2. 196 p.

Ghaly AE, Ramkumar DR, Sadaks SS, Rochon JD (2000) Effect of reseeding and pH control on the performance of a two-stage mesophilic anaerobic digester operating on acid cheese whey. Can. Agric. Eng. 42: 173-183.

Igoud S, Tou I, Kehal S, Mansouri N, Touzi A (2002) Première Approche de la Caractérisation du Biogaz Produit à Partir des Déjections Bovines. Rev. Energ. Ren. 5 : 123128. 